\title{
EchoGéo
}

$32 \mid 2015$

Inde : le grand écart spatial

\section{Le grand écart spatial de l'Inde : introduction}

Frédéric Landy

\section{OpenEdition}

Journals

Édition électronique

URL : https://journals.openedition.org/echogeo/14279

DOI : 10.4000/echogeo. 14279

ISSN : 1963-1197

\section{Éditeur}

Pôle de recherche pour l'organisation et la diffusion de l'information géographique (CNRS UMR 8586)

\section{Référence électronique}

Frédéric Landy, « Le grand écart spatial de l'Inde : introduction », EchoGéo [En ligne], 32 | 2015, mis en ligne le 15 juillet 2015, consulté le 11 août 2021. URL : http://journals.openedition.org/echogeo/14279 ; DOI : https://doi.org/10.4000/echogeo.14279

Ce document a été généré automatiquement le 11 août 2021.

EchoGéo est mis à disposition selon les termes de la licence Creative Commons Attribution - Pas d'Utilisation Commerciale - Pas de Modification 4.0 International (CC BY-NC-ND) 


\title{
Le grand écart spatial de l'Inde : introduction
}

\author{
Frédéric Landy
}

1 Le voyageur européen qui atterrit à l'aéroport international de Delhi a directement accès, par des couloirs climatisés, au métro aérien qui le relie à la ville. Une fouille rapide des bagages et des passagers filtre l'accès à la station. Une rame apparaitra rapidement, moderne, spacieuse et silencieuse. La ligne devient souterraine en arrivant dans le centre de l'agglomération. Arrivé à la station New Delhi, que notre voyageur prenne en correspondance la Yellow Line: s'il part vers le sud, le premier arrêt est Chandni Chowk, le centre d'affaires de Connaught Place. Mais qu'il choisisse le nord: le premier arrêt sera Chawri Bazaar. Notre voyageur descendra alors du wagon, prendra les escalators qui silencieusement le monteront à l'air libre. Au cours de son ascension, à mesure que le carré de lumière s'élargira au-dessus de sa tête, les bruits de la rue lui parviendront, étouffés d'abord, puis beaucoup plus sonores. Des odeurs inconnues l'interrogeront. Et puis soudain, à l'air libre, ce sera l'émergence dans une autre Inde. Celle des vendeurs de rue, des cycle rickshaws (cyclopousses), de la vieille ville musulmane aux densités de population extrêmes, de l'habitat dégradé, d'un marché urbain extrêmement dynamique où la richesse est souvent masquée aux étrangers. Notre voyageur aura changé de planète et d'époque, pour ainsi dire : à l'aéroport international comme dans le métro, il se trouvait dans un prolongement de pays du Nord ; le voici au Sud. Il se trouvait dans l'Inde du XXI ${ }^{e}$ siècle, le voici dans celle du XXe, celle de la pauvreté, du commerce informel, de pratiques urbaines et économiques qui ne correspondent pas au modèle des world-class cities. Notre voyageur ne verra pas au premier abord les changements qui ont pourtant affecté ce vieux centre, qu'il considèrera - à tort - comme frappé d'immobilisme. Il sera en revanche étonné - à juste titre - par les contrastes sociaux, culturels, économiques, spatiaux entre ce monde de l'aéroport ou du métro qu'il vient de quitter, et celui de Old Delhi.

2 L'Inde est tombée d'un cliché dans un autre : des bidonvilles et de Mère Theresa, la voilà consacrée Shining India, pays de l'informatique et de Mittal. Mais au-delà des caricatures, faut-il vraiment choisir entre ces extrêmes? Les deux aspects n'ont-ils pas 
une part de vrai, coexistant plus ou moins bien dans l'Inde " émergente» (Géoconfluences, 2015)? De fait, tout pays émergent peut sans doute se définir par un « grand écart » (Landy éd., 2010) : écart social (de plus en plus de riches, mais toujours beaucoup de pauvres), écart économique (développement de l'informatique en Inde, mais aussi du secteur informel), et écart spatial. Le présent numéro d'Echogéo est centré sur ce dernier : à l'intérieur des villes indiennes voisinent en effet bidonvilles et résidences de luxe, tandis que parallèlement se renforce la ségrégation interconfessionnelle (Sivaramakrishnan et al., 2005 ; Gayer et Jaffrelot, 2011); des régions métropolitaines se développent en laissant dans les marges les campagnes voisines; le développement d'innombrables zones franches accroît la pression sur la terre en sacrifiant agriculteurs et sécurité alimentaire à des enjeux industriels mais aussi immobiliers (Kennedy, 2010)...

3 Les inégalités spatiales et sociales s'accroissent, étant donné que les groupes et les espaces riches se multiplient, mais que ceux qui demeurent «immergés » demeurent les plus nombreux : l'Inde est bien, comme tout pays émergent, un " pays-iceberg » (Landy, Varrel, à paraître). Inégalités spatiales : le PIB par habitant du Bihar, État le plus pauvre, était en 1961 1,8 fois plus faible que celui du Maharashtra, le plus riche des grands États fédérés; mais le facteur atteignait 4,6 en 2014. Inégalités sociales : en 2009-2010, selon la National Sample Survey Organisation, le coefficient de Gini atteignait respectivement 0,28 et 0,37 en zones rurales et urbaines. Nuançons pourtant : outre que le calcul de ce coefficient est très controversé, il reste de toutes les façons très inférieur à ceux des autres pays émergents du groupe des BRICS ${ }^{1}$. Et il faut se garder de faire trop confiance à l'espace. Le géographe sait bien que les paysages peuvent être trompeurs. Les bidonvilles indiens abritent beaucoup de petites classes moyennes, et dans des villages pauvres en apparence peuvent vivre quelques familles de notables ayant une bonne part de leur capital investi dans une grande ville.

4 Les relations entre ce grand écart spatial et le processus d'émergence restent de toute façon à analyser (Durand-Dastès, 2012). Quelques hypothèses avaient été esquissées en ce sens lors de l'appel à contributions à la base de ce numéro d'Echogéo, qui ont été testées par les différents articles.

\section{Point de métropolisation concentrée sur quelques régions urbaines}

5 La métropolisation en cours du réseau urbain se traduit-elle par une perte de vitesse des petites villes et la croissance économique concentrée dans seulement quelques grandes régions urbaines ? On sait que ce n'est pas le cas (Denis, Marius-Gnanou, 2011). Les petites villes ont-elles pour autant un effet d'entraînement sur les campagnes qui permettrait de développer une diversification rurale non agricole jusqu'ici très limitée? Là encore, la réponse est plutôt négative. Dans ce numéro, C. Aubron, H. Lehoux et C. Lucas signalent que les villes du Gujarat, grandes ou petites, sont incapables de fournir assez d'emplois à la main d'œuvre rurale, relativement qualifiée ou pas. A propos de l'Assam, E. Cremin et A. Hollé ajoutent que l'accessibilité physique des ruraux aux villes est en elle-même déjà un problème, en raison des crues et des divagations $\mathrm{du}$ Brahmapoutre. Un des goulots d'étranglement de la croissance indienne, les transports, est ici mis en lumière. (Le second, l'énergie, sera analysé par N. Mazzucchi). 


\section{Des disparités même au sein des campagnes}

6 Ces grands écarts spatiaux existent-ils aussi à l'intérieur même des espaces ruraux ? Les campagnes indiennes ne demeurent-elles pas plutôt marquées par un maintien quasi uniforme de la pauvreté - les villageois riches partant en ville (Gupta, 2005) ? C. Aubron et al. montrent que même dans le Gujarat souvent présenté comme une vitrine de l'Inde en croissance, fief électoral du premier ministre Narendra Modi, il existe des espaces ruraux marqués par la pauvreté ; mais que cette pauvreté n'est pas uniforme socialement, et qu'elle est largement due à une inégale répartition de la terre et de l'eau. Alors qu'à partir du XIX $X^{e}$ siècle les Brahmanes ont quitté les campagnes de l'Inde pour s'installer en ville, libérant des terres agricoles, aujourd'hui les Patel du Gujarat comme les Kamma d'Andhra Pradesh émigrent bien vers les grandes villes indiennes ou à l'étranger, mais gardent l'essentiel de leurs terres via des contrats, de métayage souvent, qui favorisent excessivement les propriétaires et permet la dominance sur place d'une élite rurale.

\section{Ces écarts spatiaux se sont-ils tant renforcés avec « l'émergence »?}

7 Les contrastes de l'Inde sont-ils si récents, ou bien leur présence est-elle fondamentalement structurelle, ce qui rendrait plus difficile leur résorption? Après tout, F. Durand-Dastès montre bien dans ce numéro la permanence des efforts de New Delhi pour tenter, afin de réduire les écarts régionaux, de trouver des indicateurs de «retard»: d'où la définition des districts backward, premiers bénéficiaires des programmes d'aide et de développement. Les écarts préexistaient donc à l'émergence. Mais il se trouve que la croissance économique, loin de les rééquilibrer, a été la plus forte dans les zones déjà en avance. C'est ainsi que N. Mazzucchi montre que la faim d'énergie, en Inde, favorise les littoraux importateurs d'hydrocarbures, aux dépens d'une Inde orientale qui se trouve délaissée par le réseau des conduites. Comme le suggère $F$. Durand-Dastès, l'aménagement du territoire - ce que les Indiens appellent le regional planning - parvient difficilement à atteindre un niveau d'efficacité suffisant pour agir sur des différenciations profondément enracinées dans des temps longs. Par ailleurs, il existe une tendance récente à la réduction de l'ampleur de ces politiques, voire à leur abandon. E. Cremin et A. Hollé rappellent que l'existence d'aéroports relativement importants en Assam, dans le lointain Nord-Est, favorisée par une politique obligeant les lignes aériennes à les desservir, contribue à réduire l'écart entre les mégapoles de l'Inde et les villes de l'État. Mais ces flux aériens ne peuvent réduire beaucoup l'écart entre ces dernières et leur arrière-pays... Comme le suggèrent C. Aubron et al., les migrations vers la ville ne permettent pas autre chose que des revenus de survie, assurant à grand peine le maintien du reste de la famille au village mais en aucune façon son enrichissement ou une quelconque capitalisation. En cela leur article atteste du cas d'école du Gujarat : voici l'État qui a la plus forte croissance agricole du XXI siècle, doté d'une puissance industrielle remarquable... mais qui ne parvient pas à assurer la sécurité alimentaire de toute sa population. On ne voit alors que trois solutions pour les campagnes du Gujarat, et sans doute de l'Inde: la rémunération des services environnementaux produits par l'agriculture (biodiversité, 
écotourisme...); la diversification économique au profit d'activités non agricoles vraiment rémunératrices; ou bien, comme $C$. Aubron et al. le suggèrent, la poursuite de véritables réformes agraires et d'un développement agricole fondé sur la petite exploitation (« révolution blanche » du lait). Autant de fenêtres, mais difficiles à ouvrir largement...

\section{La pauvreté comme un des fondements de la compétitivité}

8 Les poches de pauvreté qui demeurent « immergées » sont-elles des héritages du passé, simples kystes non encore résorbés par le processus de développement, ou bien au contraire des éléments essentiels à «l'émergence » même? Le secteur informel, loin d'être un monde "archaïque ", ne permet-il pas la compétitivité des entreprises exportatrices qui travaillent avec lui ? De même que le livret hukou a permis de bâtir la croissance des villes orientales de Chine avec une main-d'œuvre bon marché, puisque presque sans droits sociaux, les slums indiens sont peut-être le moyen de faire fonctionner à bas coût Bombay ou Delhi. Doit-on vraiment parler d'« exclusion " pour des habitants de bidonvilles dont seulement 1 ou $2 \%$ sont des mendiants (SaglioYatzmirsky, Landy éd., 2013) ? On voit bien l'enjeu politique d'une telle question. L'émergence est-elle un processus favorisant à terme la convergence, ou bien repose-telle sur le maintien des inégalités? Comme se le demande E. Leclerc, "émergence mondiale et divergence nationale sont-elles compatibles? » Si la croissance repose sur les inégalités, elle se trouve éminemment fragile, avec des conséquences possibles sur le fonctionnement de "la plus grande démocratie du monde». Sur le long terme, si l'Inde ne veut pas connaître de graves problèmes politiques, il semble bien qu'elle soit condamnée à devoir générer des processus de " convergence » régionale aussi bien que sociale.

9 Non cependant que le bilan soit entièrement noir: ainsi, E. Leclerc insiste sur l'importance des espaces et des relations "hybrides", et se refuse de parler exclusivement en termes de dichotomie ou d'éclatement urbain. Même une technopole comme Cyberabad, aux côtés parfois "californiens", se trouve bien intégrée dans l'agglomération d'Hyderabad dont elle fait fonctionnellement et morphologiquement partie. E. Ponceaud-Goreau montre quant à elle, dans son étude des écoles maternelles au Tamil Nadu et à Pondichéry, que la libéralisation a permis une explosion des établissements privés qui, sans être tous de qualité ni bon marché, ont concouru à l'amélioration générale de la scolarisation. Ces écoles suivent l'étalement urbain en s'installant dans les nouveaux quartiers ou les petites villes gagnées par la métropolisation. L'État, traditionnellement très actif dans ces régions méridionales mais lucide sur les limites de ses propres capacités, encourage l'extension du secteur privé en le subventionnant. L'énorme investissement éducatif des familles aura-t-il pour conséquence à terme une meilleure formation de la population indienne, ce qui permettrait une croissance économique fondée sur des bases plus solides qu'une compétitivité reposant trop souvent sur de bas salaires? Ou bien ces personnes diplômées viendront-elles renforcer les bataillons du chômage et du sous-emploi, déjà très importants? 


\section{Un nouveau modèle de ville « postmoderne »?}

10 On pourrait se demander si l'Inde - comme peut-être d'autres pays - n'est pas en train d'inventer un nouveau modèle "postmoderne» d'agglomérations, composé de contrastes et d'informalité: des morphologies spatiales et sociales qu'en raison notamment de «l'oubli des villes de l'Inde " (Louiset, 2011), l'aménagement urbain usuel, les décideurs mais aussi certains chercheurs continuent de considérer comme «marginales» et donc susceptibles de disparaître. Les articles de C. Guéguen et d'E. Leclerc sur Kolkata et Hyderabad donnent quelques pistes sur le fonctionnement parfois « chaotique » de ces villes à propos desquelles on pourrait dire : «Et pourtant, elles tournent ». Pourtant, un regard historique même minime oblige à s'interroger sur les termes mêmes du débat. Chandigarh, planifiée notamment par Le Corbusier, et toutes les «villes nouvelles » d'une certaine génération, de New Delhi à Gandhinagar, apparaissent des exceptions où la vision aménagiste et hygiéniste était relayée par un pouvoir politique si puissant que les structures de ces villes ne sont pas loin, encore aujourd'hui, de fonctionner selon les normes occidentales. Pour le reste, les villes indiennes, dans leur passé de villes islamiques, de villes-temples, de villes-bazar, n'ont jamais reflété les canons des urbanistes - qu'ils soient étrangers ou indiens. Aussi E. Leclerc peut-il refuser le terme de "postmoderne" pour la simple raison que, résume-il en paraphrasant Latour, « la ville indienne n'a jamais été moderne ».

\section{Le brouillage des échelles}

11 Mais la postmodernité, ne serait-ce pas plutôt, si l'on raisonne verticalement, un retrait unilatéral de l'État (fédéral et fédéré) au profit des niveaux de pouvoir décentralisés et mondialisés? Depuis la fin des années 1980 en effet, l'Inde a connu deux mouvements parallèles (et nullement contradictoires, bien au contraire): libéralisation économique - consacrée par les réformes de 1991 - et décentralisation avec les amendements constitutionnels de 1992. Le vieil État nehruvien, qui voulait promulguer "modernité » et "développement », n'est-il pas complètement disloqué ? Nos articles suggèrent que ce n'est pas le cas. Il apparaît que se produit, dans le cadre d'un jeu qui n'est pas à somme nulle, une multiplication des acteurs laissant toute sa place à l'État, dans le cadre d'un repositionnement (rescaling) complexe des modes de gouvernance (Kennedy, 2013 ; Jenkins et al., 2014)). En fait, le " grand écart » n'est pas toujours favorisé par la libéralisation économique et l'ancrage dans la mondialisation, notamment parce que New Delhi est loin de toujours laisser la bride sur le cou aux États fédérés. L. Kennedy le démontre dans ce numéro en analysant la politique des zones franches, qui permet au gouvernement fédéral de regagner par la bande un pouvoir de contrôle sur les États: des préfets (" commissaires du développement ») sont chargés de participer à la gestion des zones, dans le cadre d'« une stratégie politique pour contourner les gouvernements régionaux, jugés incapables par New Delhi de répondre à ses attentes en termes de performance économique ». Ce brouillage des échelles dans le cadre d'une ouverture au monde est également visible, quoique plus en filigrane, avec l'analyse de la réhabilitation du chinatown de Kolkata/Calcutta par C. Guéguen : ici, des États-nations (la Chine, Singapour, l'Inde) interagissent de concert ou en tension avec des communautés transnationales (la diaspora chinoise), un État fédéré (le Bengale occidental) et une municipalité (Kolkata), pour patrimonialiser quelques 
quartiers urbains. Une seule échelle est finalement assez peu représentée ici : celle de la population locale, à qui on semble peu demander son avis.

12 Or, il faut rappeler que les disparités à fine échelle locale sont beaucoup plus fortes qu'à l'échelle macro. Il y a moins de différence de revenu entre le Bihar et Goa qu'entre un habitant de bidonville et son voisin d'une résidence huppée à Mumbai/Bombay. Assurément, une telle relation est validée dans tous les pays du monde : les effets de zoom avant grossissent toujours les différences. Mais ces effets d'échelles sont particulièrement prégnants en Inde, si on la compare par exemple au Brésil ou à la Chine où les inégalités régionales sont plus fortes. Le grand écart demeure relativement peu marqué à l'échelle nationale, mais il est béant à l'échelle locale.

\section{BIBLIOGRAPHIE}

Denis D., Marius-Gnanou K., 2011. Toward a better appraisal of urbanization in India. Cybergeo, article 569 : http://cybergeo.revues.org/24798

Durand-Dastès F., 2012. La population indienne en 2011: anciennes et nouvelles différenciations spatiales. Mappemonde, 108, http://mappemonde.mgm.fr/num36/articles/art12401.html Gayer L., Jaffrelot C. (éd.), 2011. Muslims in Indian Cities: Trajectories of Marginalisation. Londres, Hurst.

Géoconfluences, 2015. Dossier en ligne sur le monde indien http://geoconfluences.ens-lyon.fr/ informations-scientifiques/dossiers-regionaux/le-monde-indien-populations-et-espaces Gupta D., 2005. Whither the Indian village ? Economic and Political Weekly, 19 février, p. 751-758. Jenkins R., Kennedy L., Mukhopadhyay P. éd., 2014. Power, Policy, and Protest: The Politics of India's Special Economic Zones. New Delhi: Oxford University Press, 396 p.

Kennedy L., 2010. La politique contestée des zones économiques spéciales en Inde. Communication Réseau Asie http://www.reseau-asie.com/edito/zones-economiques-speciales-zes-inde/

Kennedy L., 2013. The Politics of Economic Restructuring in India: Economic Governance and State Spatial Rescaling. Londres, Routledge, $192 \mathrm{p}$.

Landy F. (éd.), 2010. Dictionnaire de l'Inde contemporaine. Paris, Armand Colin, 566 p.

Landy F., Varrel A., à paraître. L'Inde. Paris, Armand Colin, collection U.

Louiset O., 2011. L'oubli des villes de l'Inde. Pour une géographie culturelle de la ville. Armand Colin, $296 \mathrm{p}$.

Saglio-Yatzimirsky M.C., Landy F. (éd.), 2013. Megacity Slums: Social Exclusion, Space and Urban Policies in Brazil and India. Londres, Imperial College Press, $446 \mathrm{p}$.

Sivaramakrishnan K.C., Kundu A., Singh B.N., 2005. Handbook of Urbanisation in India. New Delhi, Oxford University Press, 195 p. 


\section{NOTES}

1. Voir par exemple http://data.worldbank.org/indicator/SI.POV.GINI

INDEX

Thèmes : Sur le Champ - Sur le Terrain

\section{AUTEUR}

\section{FRÉDÉRIC LANDY}

Frédéric Landy, frederic_landy@orange.fr, est Professeur des universités, Directeur du département de géographie de l'Université Paris Ouest-Nanterre, membre du laboratoire Mosaïque, UMR LAVUE et membre associé du Centre d'Etudes de l'Inde et de l'Asie du Sud (CNRSEHESS). Il a publié récemment :

- Landy F., Varrel A., à paraître en septembre 2015. L'Inde. Du développement à l'émergence. Armand Colin.

- Landy F., Moreau S., 2014. Le droit au village/The right to the village. Justice spatiale/Spatial Justice, 7, en ligne www.jssj.org

- Landy F., Saglio-Yatzimirsky M.C., 2013. Megacity slums. Social Exclusion, Space and Urban Policies in Brazil and India. Imperial College Press, $464 \mathrm{p}$. 